Research Article

\title{
Effects of High-Density Electric Current Pulse on the Undercooling of Fe-B Eutectic Alloy Melt
}

\author{
Teng Ma $\mathbb{D}^{1},{ }^{1}$ Weixin Hao $\mathbb{D}^{1},{ }^{1}$ Xiaosi Sun, ${ }^{1}$ Junting Zhang, ${ }^{2}$ and Guihong Geng $\mathbb{D}^{3}$ \\ ${ }^{1}$ School of Materials Science and Engineering, Taiyuan University of Science and Technology, Taiyuan 030024, China \\ ${ }^{2}$ School of Applied Science, Taiyuan University of Science and Technology, Taiyuan 030024, China \\ ${ }^{3}$ School of Materials Science and Engineering, North Minzu University, Yinchuan 750000, China \\ Correspondence should be addressed to Weixin Hao; wxhao@vip.sina.com and Guihong Geng; gengguihong@163.com
}

Received 12 December 2017; Revised 17 January 2018; Accepted 24 January 2018; Published 23 April 2018

Academic Editor: Yoshitake Masuda

Copyright ( $) 2018$ Teng Ma et al. This is an open access article distributed under the Creative Commons Attribution License, which permits unrestricted use, distribution, and reproduction in any medium, provided the original work is properly cited.

\begin{abstract}
The solidification microstructure of Fe-B eutectic alloy under high undercooling and high-density electric current pulse (ECP) was investigated with the technique of molten glass slag purification combined with cyclical superheating and the ECP treatment. The effects of high-density ECP on the undercooling of Fe-B eutectic alloy melt were analyzed by the DSC method. The analysis results showed that the solidification microstructure of Fe-B eutectic alloy under ECP was similar to that obtained by the high undercooling technique. The undercooling obtained under two experimental conditions was basically the same, proving that the high undercooling of the metallic melt could be realized by the ECP.
\end{abstract}

\section{Introduction}

High undercooling refers to a phenomenon that crystallization or solidification does not occur when a liquid metal is cooled below the liquids. Eutectic alloy is widely used as the casting alloy, and great progresses have been made in the studies in undercooling of eutectic alloy. Many experimental phenomena, such as refinement of solidification structure, reduction of the segregation, improvement of the distribution of impurity, the formation of metastable phase and amorphous phase, the growth transformation of eutectic alloy, and soluting trapping [1-5], had been found. Undercooling is an important parameter in the solidification process of metals and metal melts.

As a new type of green solidification control technology, the ECP, which conducted in alloys through electrodes directly contacting the melt, can improve the heterogeneous nucleation rate of liquid metal or semi-liquid metal, promote the solute redistribution, and refine the solidification microstructure [6-9]. Many research studies have been carried out to investigate the effect of ECPs on the solidification of pure metals [10], eutectic alloys [11-13], and solid solution alloys [14] in the past decades. The mechanisms proposed to understand the
ECP-induced grain refinement include the heterogeneous nucleation mechanism that the nucleation rate is promoted owing to the raised undercooling $[7,15,16]$, the skin effect, the dendrite fragmentation mechanism caused by Joule heating [17], Lorenz force [11, 15], and the crystal rain [10].

The effect of ECP on the undercooling of the melt had been confirmed by many scholars [7, 16, 18]. Previous studies on the undercooling of ECP were performed under the condition of large voltage $[19,20]$, but the undercooling of ECP under low voltage was not studied. In the study, with $\mathrm{Fe}_{83} \mathrm{~B}_{17}$ eutectic alloy as the research object, by means of molten glass slag purification and cyclical superheating, according to the high-density ECP method, the influences of the undercooling on solidification microstructure of metallic melt were studied. The undercooling variation of alloy melt was detected by DSC method and explained theoretically. The ECP as a novel solidification technology can be applied in engineering practice.

\section{Experimental Methods}

2.1. Selection of Alloy. The Fe-B alloy is a kind of good soft magnetic material possessing excellent glass forming ability 


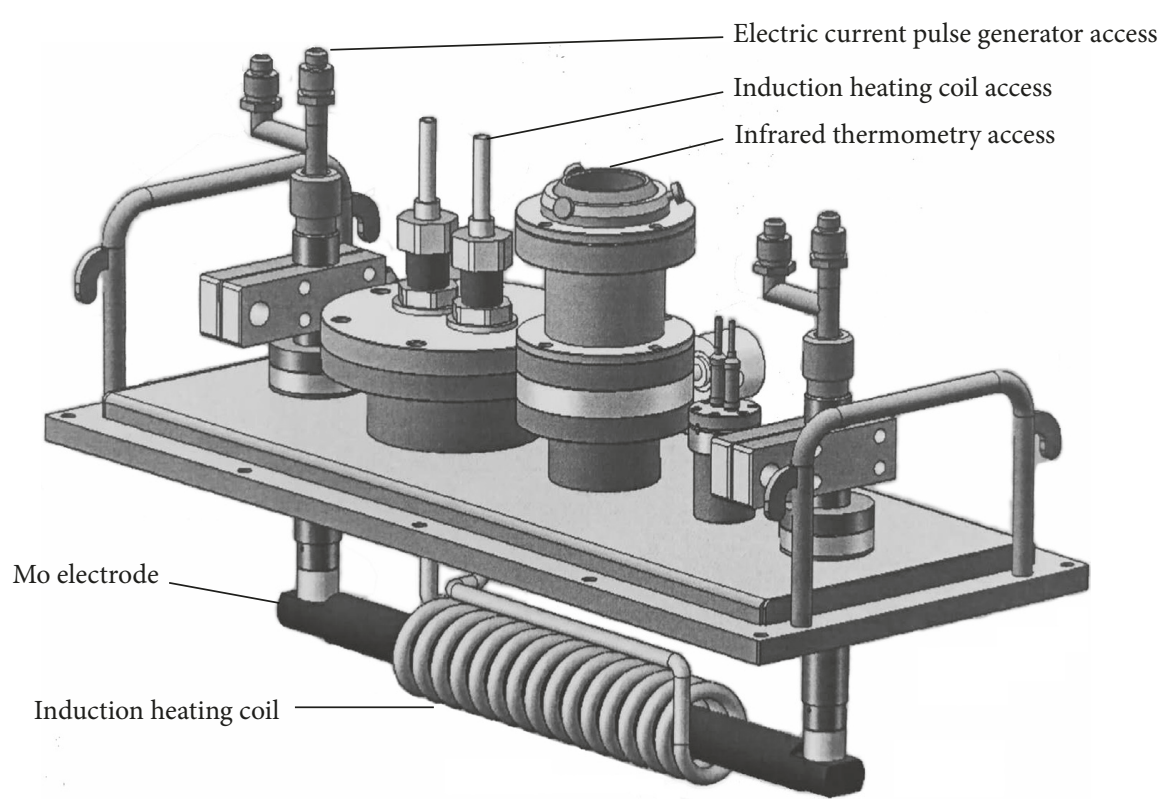

Figure 1: Schematic drawing of experimental setup.

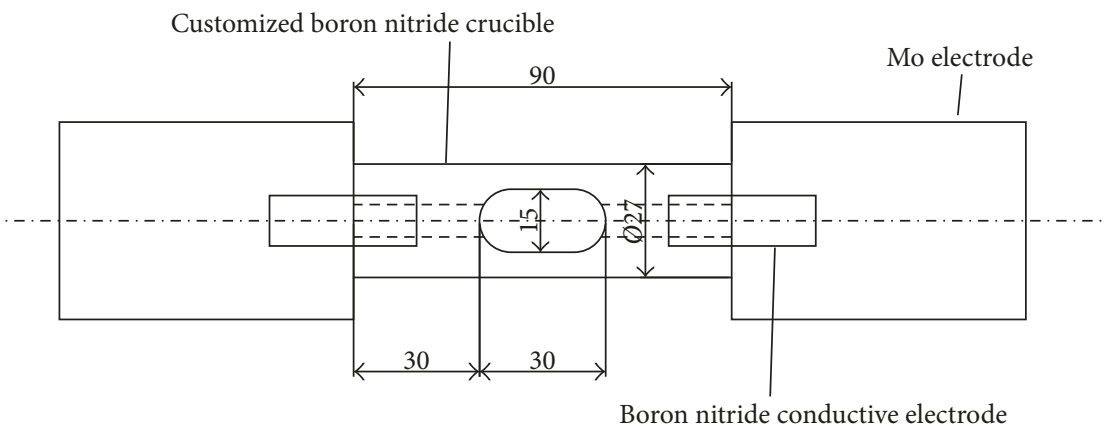

FIGURE 2: Schematic sketch of the crucible and the eletrodes.

in iron-rich end and can realize the greater undercooling [21]. With the eutectic alloy $\mathrm{Fe}_{83} \mathrm{~B}_{17}$ as the research object, the solidification evolutions and the undercooling were discussed respectively under the conditions of high undercooling condition and high-density ECP.

2.2. Preparation of Undercooled FeB Alloy. The alloy was melted and purified utilizing the high frequency induction heating device (Fe, B purity was above $99.99 \%$ ). The output power was $35 \mathrm{~kW}$, and the working frequency was $20 \sim 50 \mathrm{kHz}$. The diameter of induction heating copper coil was $6 \mathrm{~mm}$. The molten glass slag purification and cyclical superheating were used to purify molten metals with $\mathrm{B}_{2} \mathrm{O}_{3}$ purification. In the experiment, the temperature of alloy melt measured by infrared thermometer was output to the connected computer through the active RS485 converter, and the measured data were stored.

2.3. Preparation of FeB Alloy under High-Density ECP. Figure 1 is the schematic drawing of experimental setup. The experimental apparatus consist of the customized pulse power supply, vacuum system, high frequency induction heating device, and temperature measuring system. Figure 2 is schematic sketch of the crucible and the electrodes. The pulse power provides a frequency from 0 to $50 \mathrm{~Hz}$, and the electric voltage ranges from 0 to $100 \mathrm{~V}$. First, the master alloy was prepared according to the proportion of eutectic components. The $\mathrm{Fe}_{83} \mathrm{~B}_{17}$ master alloy was cut into the specimens with the size of $15 \mathrm{~mm} \times 10 \mathrm{~mm} \times 15 \mathrm{~mm}$, put into a customized boron nitride crucible with a cylindrical cavity, and covered with $\mathrm{B}_{2} \mathrm{O}_{3}$. The boron nitride conductive electrode (chemical composition: $\mathrm{BN}+\mathrm{TiB}_{2}+\mathrm{AIN}$ ), connected to the crucible with the Mo electrodes, was used in the ECP treatment with thermal conductivity $100 \mathrm{~W} / \mathrm{mK}$ to reducing the heterogeneous nucleation. The samples were melted in the crucible and headed to $1500^{\circ} \mathrm{C}$ in $\mathrm{N}_{2}$ atmosphere. The ECP treatment was performed when the end face of boron nitride electrode contacted the molten metal horizontally according to the parameters of pulse voltage $20 \mathrm{~V}$, pulse frequency $30 \mathrm{~Hz}$, pulse width $20 \mu$ s, peak current $600 \mathrm{~A}$, and pulse current duration of $30 \mathrm{~s}$. The cooling rate used was $10 \mathrm{~K} / \mathrm{min}^{-1}$, which was held approximately constant throughout the solidification. After cooling, the $10 \mathrm{~mm}$ piece test sample was cut from solidified 


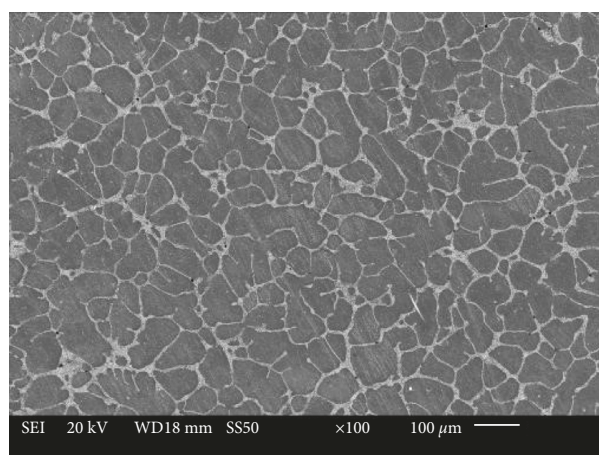

(a)

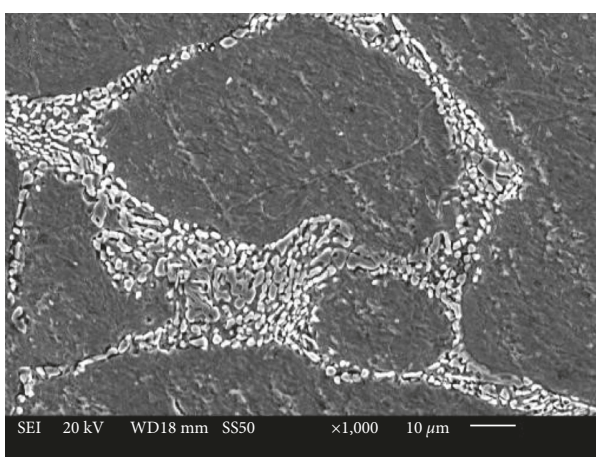

(c)

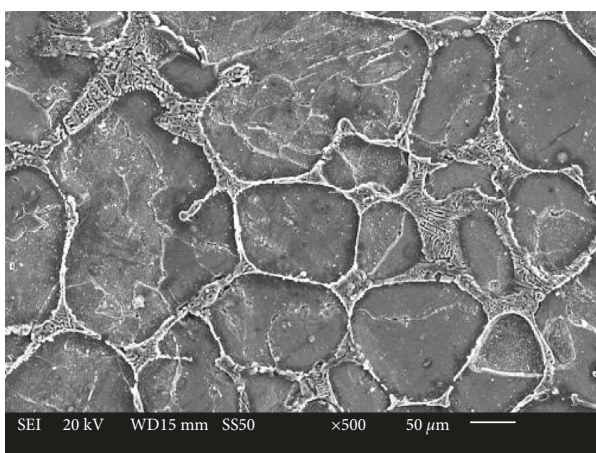

(e)

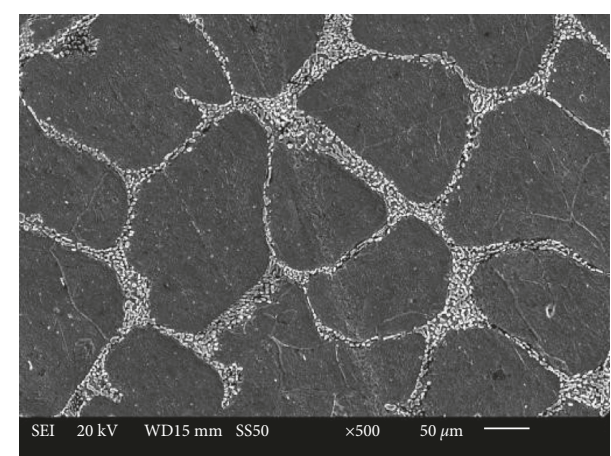

(b)

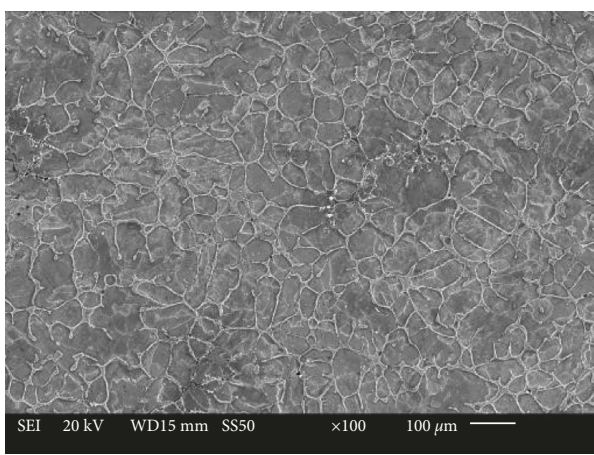

(d)

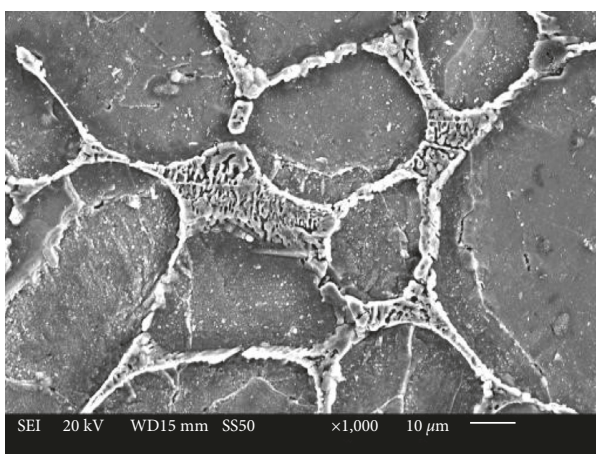

(f)

FIGURE 3: Solidification structure of the sample under the high undercooling conditions and ECP treatment. (a)-(c) Solidification structure of the sample under the high undercooling. (d)-(f) Solidification structure of the sample treated with the ECP.

specimen and then polished for metallographic examination. The etching reagent used to reveal the macrostructure was nitric acid. The microstructures were observed by SEM along the center line of the specimen.

\section{Results and Discussion}

Figures 3(a)-3(c) show the hypereutectic microstructure of $\mathrm{Fe}_{83} \mathrm{~B}_{17}$ eutectic alloy under high undercooling conditions. According to the Fe-B equilibrium phase diagram, X-ray powder diffraction spectrum (Figure 4) and the previous studies indicated the primary phase of $\alpha$-Fe (shown as dark grey phase) and the irregular eutectic of $\alpha-\mathrm{Fe}+\beta\left(\mathrm{Fe}_{2} \mathrm{~B}\right)$ (shown as light grey phase) forming the noncontinuous network distributed in the grain boundary between $\alpha$-Fe. Figures 3(d)-3(f) show the microstructures of the intermediate alloy treated with the ECP. Similar grain sizes and microstructures were obtained by ECP compared with the ones by high undercooled technology. As shown in Figures 3(c) and 3(f), the volume fraction of the eutectic phase $\alpha-\mathrm{Fe}+\beta\left(\mathrm{Fe}_{2} \mathrm{~B}\right)$ is less decreased. Figure 5 shows that the lamellar regular eutectic structure of original FeB sample prepared without undercooling and ECP is different from the hypereutectic microstructure of the sample under the high undercooling conditions and ECP treatment.

It has been proposed that the maximum undercooling can be raised to accelerate the heterogeneous nucleation rate. Solidification behavior under high undercooling is an extremely nonequilibrium solidification behavior, which produces the primary phase and the eutectic phase. It was proved by many experiments that the binary eutectic alloy with a facet became an irregular eutectic microstructure when the undercooling reached or exceeded a critical value [22, 23]. With the heating 


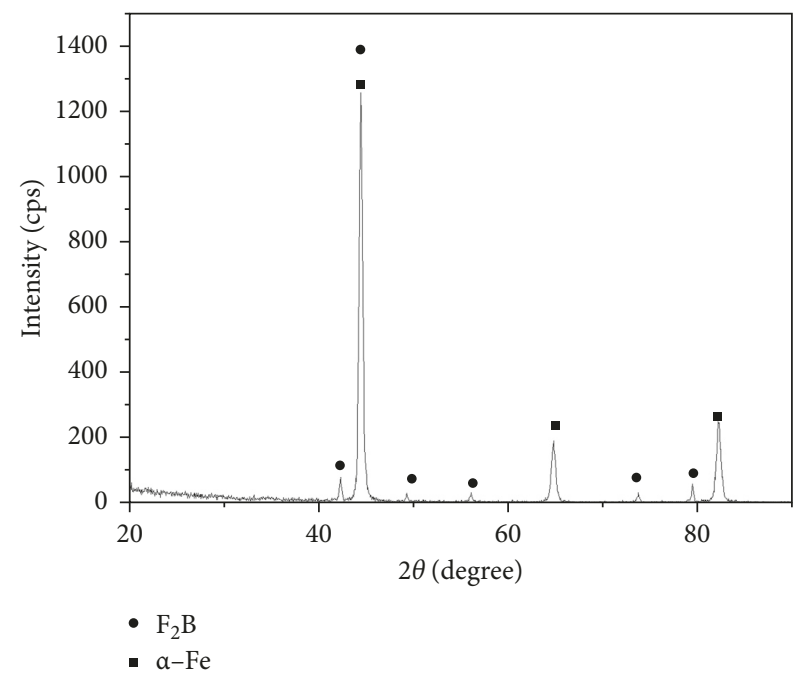

Figure 4: X-ray powder diffraction spectrum of $\mathrm{Fe}_{83} \mathrm{~B}_{17}$ eutectic alloy.

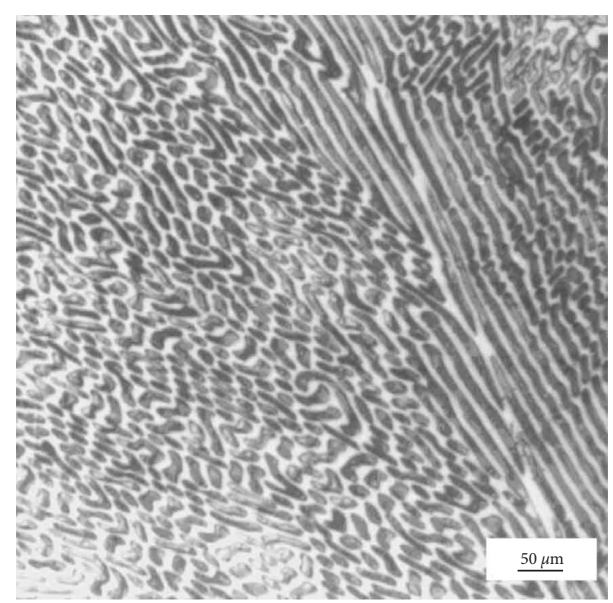

Figure 5: Solidification structure of $\mathrm{Fe}_{87} \mathrm{~B}_{13}$ sample prepared without undercooling and ECP treatment.

and cooling rate of $10 \mathrm{~K} / \mathrm{min}^{-1}$, the DSC curve of undercooled melt $\mathrm{Fe}_{83} \mathrm{~B}_{17}$ (Figure 6) shows that the undercooling degree of melt alloy is $95 \mathrm{~K}$. In the solidification process, there are two recalescence peaks because the latent heat release rate of phase change is much higher than heat dissipation. The solidification process is divided into two stages: the precipitation of $\alpha$-Fe as the primary phase and the eutectic reaction of $\mathrm{L} \rightarrow \alpha-\mathrm{Fe}+\beta\left(\mathrm{Fe}_{2} \mathrm{~B}\right)$ of the residual liquid phase. The two recalescence peaks on the temperature curve correspond to the solidification of two phases. In Figure 3(c), the $\alpha$-phase in $\alpha$-Fe $+\beta\left(\mathrm{Fe}_{2} \mathrm{~B}\right)$ between the grain boundaries grows and is attached to the primary phase $\alpha$-Fe into one because the solute trapping caused by nonuniform solute atom diffusion leads to the increase of the content of $\alpha$-Fe in the residual liquid phase and the inhomogeneity of the eutectic structure.

Compared with the solidification process under undercooling conditions, the morphology affected by ECP is similar but the structure is more dense and uniform. In the

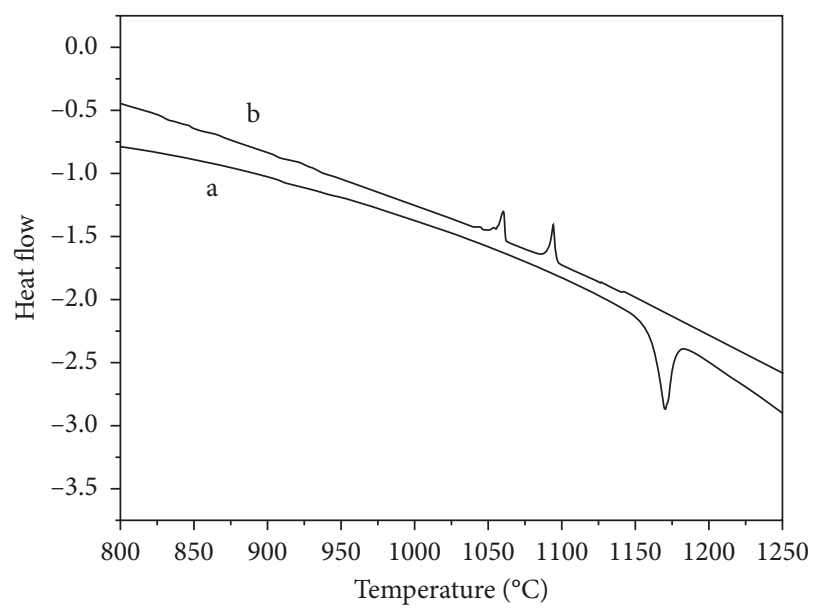

Figure 6: DTA traces for $\mathrm{Fe}_{83} \mathrm{~B}_{17}$ heating (curve a) and cooling (curve b) runs were performed at a rate of $10 \mathrm{~K} \cdot \mathrm{min}^{-1}$.

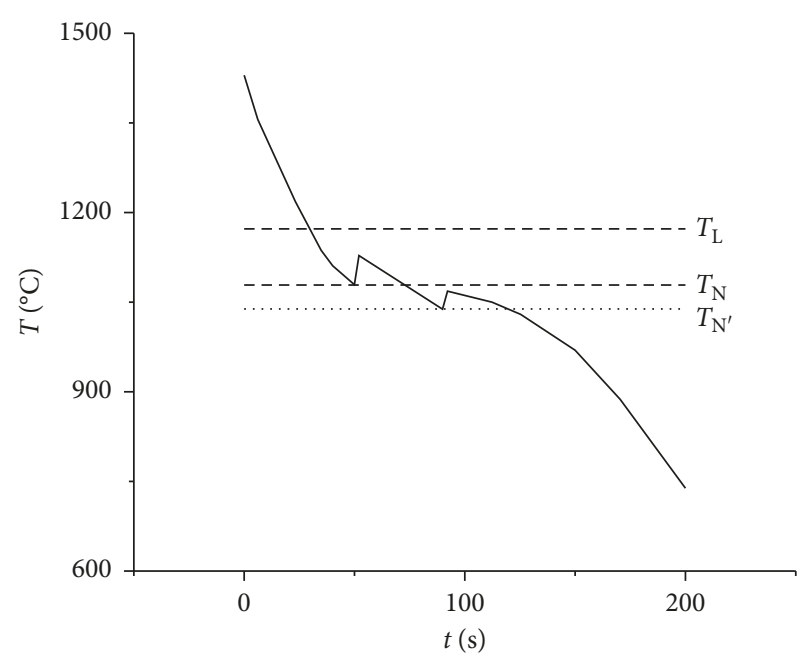

FIgURE 7: Typical cooling curves of $\mathrm{Fe}_{83} \mathrm{~B}_{17}$ in nonequilibrium solidification conditions.

metal melt, $\alpha$-Fe primary phase recalesces rapidly after nucleation and growth. Due to the thermal shock of recalescence, the dendrite of $\alpha$-Fe fusing and equiaxed grains were formed. The above-mentioned dendritic fragments were ripened and surrounded by eutectic phase $\alpha-\mathrm{Fe}+\beta\left(\mathrm{Fe}_{2} \mathrm{~B}\right)$ precipitated in residual melts, thus forming the irregular eutectic. The cooling curve (Figure 7) shows two recalescence peaks. The undercooling of alloy melt under highdensity ECP is $105 \mathrm{~K}\left(\Delta T=T_{\mathrm{L}}-T_{\mathrm{N}}, \quad T_{\mathrm{L}}=1173^{\circ} \mathrm{C}\right.$, $T_{\mathrm{N}}=1068^{\circ} \mathrm{C}$ ), which was basically the same to that obtained under high undercooling conditions.

\section{Mechanism Explanation}

The above results showed that similar solidification microstructures were obtained under high-density ECP and high undercooling technique. The cooling curve indicated that the same undercooling could be obtained by highdensity pulse current and high undercooling technique. In this paper, it is proved that the melt undercooling can be 
improved by high-density ECP. The mechanism is interpreted as follows:

(1) According to the atomic cluster theory proposed by Wang [24], the ECP as a kind of energy access cracked large clusters of atoms into smaller clusters. Therefore, the number of small clusters in the same volume of melts increased to strengthen the surface energy of clusters. As a result, higher undercooling was required to provide the nucleation energy for increasing the size of the smaller clusters to the critical nucleation radius. Therefore, the ECP increased the molten metal undercooling. The clusters' sizes decreased, and a larger number of clusters were required for meeting the critical nucleation radius to gain more grains. In this way, the alloy was refined.

(2) The dendrite fragmentation was another origin for grain refinement under the application of ECP. It is possible that the forced flow can trigger the remelting of high-order dendrite arms to detach from the dendrite trunk due to the forced flow-induced solute fluctuation [13]. The detached dendrite arms would be subsequently transported out as new potential nuclei sites under the influence of forced flow. Since the size of dendrite arms is really less than the maternal dendrite, numerous tiny grains mixed with some larger grains are consequently formed.

(3) The skin effect associated with the current pulses raised the temperature of the molten metal near the mold wall, thereby suppressing the heterogeneous nucleation. Then, the undercooling increased. The skin depth $\delta$ of a current pulse is given by

$$
\delta=\sqrt{\frac{\rho}{\pi \mu f}},
$$

where $\rho$ is resistivity of the molten alloy; $\mu$ is permeability; and $f$ is the electric pulse current wave frequency [7].

(4) The crystal grew in the crucible, so the performance and quality of the crucible played an important role in the quality of crystal growth. The thermal conductivity, thermal expansion coefficient, wetting angle, surface finish, chemical stability, and purity of the crucible determined the structures and properties of the crystal. The crucible used in this experiment was a kind of high-purity boron nitride material, which was better than the traditional quartz crucible. The crucible used in this experiment showed the characteristics of small thermal expansion coefficient, high thermal conductivity, obvious anisotropy, and large wetting angle and can eliminate more heterogeneous nucleation and obtain large undercooling degree of the melt.

\section{Conclusions}

(1) The similar grain sizes and the same microstructures of $\mathrm{Fe}_{83} \mathrm{~B}_{17}$ alloy were obtained by high-density ECP and high undercooled technology. The $\alpha$-Fe is the primary phase, and the irregular eutectic $\alpha$-Fe $+\beta\left(\mathrm{Fe}_{2} \mathrm{~B}\right)$ formed the noncontinuous network distributed in the grain boundary between $\alpha$-Fe. The eutectic phase of $\alpha$-Fe + $\beta\left(\mathrm{Fe}_{2} \mathrm{~B}\right)$ became finer and the volume fraction was decreased.

(2) DSC method indicated that the similar undercooling of alloy melt was obtained under high-density ECP and high undercooling. It was proved that the high undercooling applied on the metallic melt could be realized by the ECP, thus laying the foundation for large-scale production practice.

(3) The atom cluster cracking, the dendrite fragmentation, the skin effect, and the crucible material are responsible for the melt undercooling increased by high-density ECP.

\section{Conflicts of Interest}

The authors declare that there are no conflicts of interest regarding the publication of this paper.

\section{Acknowledgments}

This work was supported by the National Natural Science Foundation of China (51561001, 51574171, and 51641406) and the Natural Science Foundation of Shanxi Province of China (no. 201601D011012).

\section{References}

[1] J. H. Perepezko, "Nucleation reactions in undercooled liquids," Materials Science and Engineering A, vol. 178, no. 1-2, pp. 105111, 1994.

[2] Y. Lu, F. Liu, G. Yang, H. Wang, and Y. Zhou, "Grain refinement in solidification of highly undercooled eutectic Ni-Si alloy," Materials Letters, vol. 61, no. 4-5, pp. 987-990, 2007.

[3] B Wei, D. M. Herlach, and F. Sommer, "Rapid eutectic growth of undercooled metallic alloys," Journal of Materials Science Letters, vol. 12, no. 22, pp. 1774-1777, 1993.

[4] L. Battezzati, C. Antonione, and M. Baricco, "Undercooling of $\mathrm{NiB}$ and $\mathrm{FeB}$ alloys and their metastable phase diagrams," Journal of Alloys and Compounds, vol. 247, no. 1-2, pp. 164-171, 1997.

[5] J. Hua, Y. Zhang, and C. Wu, "Grain refinement of $\mathrm{Sn}-\mathrm{Pb}$ alloy under a novel combined pulsed magnetic field during solidification," Journal of Materials Processing Technology, vol. 211, no. 3, pp. 463-466, 2011.

[6] B. T. Zi, K. F. Yao, W. J. Liu, J. Z. Cui, and Q. X. Ba, "Effect of higher density pulsed current on solidification structures of $2024 \mathrm{Al}$ alloy," Rare Metal Materials and Engineering, vol. 32, no. 1, pp. 9-12, 2003.

[7] J. P. Barnak, A. F. Sprecher, and H. Conrad, "Colony (grain) size reduction in eutectic $\mathrm{Pb}-\mathrm{Sn}$ castings by electroplusing," Scripta Metallurgica Et Materialia, vol. 32, no. 6, pp. 879-884, 1995.

[8] S. X. He, J. Wang, B. D. Sun, and X. H. Zhou, "Effect of high density pulse current on solidification structure of hypereutectic Al-19Si alloy," Chinese Journal of Nonferrous Metals, vol. 12, no. 2, pp. 275-278, 2005.

[9] H. Y. Sun, D. J. Lv, W. Li, and Y. W. Jiang, "Impact of electric pulses to structure and mechanical properties of common 
metal and high specific gravity tungsten alloy," Journal of Harbin University of Commerce, vol. 27, no. 5, pp. 733-740, 2011.

[10] X. Liao, Q. Zhai, J. Luo, W. Chen, and Y. Gong, "Refining mechanism of the electric current pulse on the solidification structure of pure aluminum," Acta Materialia, vol. 55, no. 9, pp. 3103-3109, 2007.

[11] D. Rabiger, Y. Zhang, V. Galindo, S. Franke, B. Willers, and S. Eckert, "The relevance of melt convection to grain refinement in Al-Si alloys solidified under the impact of electric currents," Acta Materialia, vol. 79, pp. 327-338, 2014.

[12] Y. Zhou, S. Xiao, and J. Guo, "Recrystallized microstructure in cold worked brass produced by electropulsing treatment," Materials Letters, vol. 58, no. 12-13, pp. 1948-1951, 2004.

[13] Z. Lu, C. Guo, P. Li et al., "Effect of electropulsing treatment on microstructure and mechanical properties of intermetallic Al3Ti alloy," Journal of Alloys and Compounds, vol. 708, pp. 834-843, 2017.

[14] S. Ahmed and E. C. Mckannan, "Control of $\gamma^{\prime}$ morphologyin nickel base superalloys through alloy design and densification processing under electric field," Materials Science and Technology, vol. 10, pp. 941-946, 1994.

[15] M. Nakada, Y. Shiohara, and M. C. Flemings, "Modification of solidification structures by pulse electric discharging," ISIJ International, vol. 30, no. 1, pp. 27-33, 1990.

[16] Z. Zhao, J. Wang, and L. Liu, "Grain refinement by pulse electric discharging and undercooling mechanism," Materials and Manufacturing Processes, vol. 26, no. 2, pp. 249-254, 2011.

[17] H. Z. Liu, Z. L. Zhao, and L. H. Ma, "Influence of high density pulse electric current on solidified aluminum structure and undercooling degree," Foundry Technology, vol. 29, no. 10, pp. 1354-1358, 2008.

[18] F. Balima, F. Bellin, and D. Michau, "High pressure pulsed electric current activated equipment (HP-SPS) for material processing," Materials and Design, vol. 139, pp. 541-548, 2018.

[19] X. Ye, J. Kuang, X. Li, and G. Tang, "Microstructure, properties and temperature evolution of electro-pulsing treated functionally graded Ti-6Al-4V alloy strip," Journal of Alloys and Compounds, vol. 599, pp. 1-9, 2014.

[20] S. X. He, J. Wang, and X. H. Zhou, "Effect of high density pilse electric current on the solidification structure of low temperature melt of A356 aluminium alloy," Acta Metallurgica Sinica, vol. 38, no. 5, pp. 479-482, 2002.

[21] C. L. Yang, G. C. Yang, Y. P. Lu, Y. Z. Chen, and Y. H. Zhou, "Phase selection in highly undercooled Fe-B eutectic alloy melts," Transactions of Nonferrous Metals Society of China, vol. 16, no. 1, pp. 39-43, 2006.

[22] L. Q. Xing, D. Q. Zhao, and X. C. Chen, "Solidification of undercooled Ni-32.5wt\%Sn eutectic alloy," Journal of Materials Science, vol. 28, no. 10, pp. 2733-2737, 1993.

[23] B. L. Jones, "Growth mechanisms in undercooled eutectics," Metallurgical Transactions, vol. 2, no. 10, pp. 2950-2951, 1971.

[24] J. Z. Wang, The Research of Treating Technology with Electropulse Modification and the Hypothesis of Liquid Metal Cluster Structure, Ph.D. dissertation, University of Science and Technology Beijing, Beijing, China, 1998. 


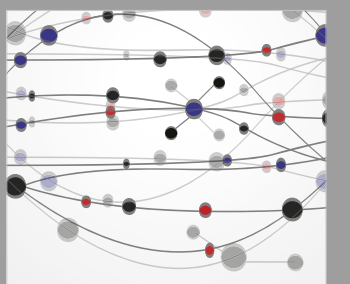

The Scientific World Journal
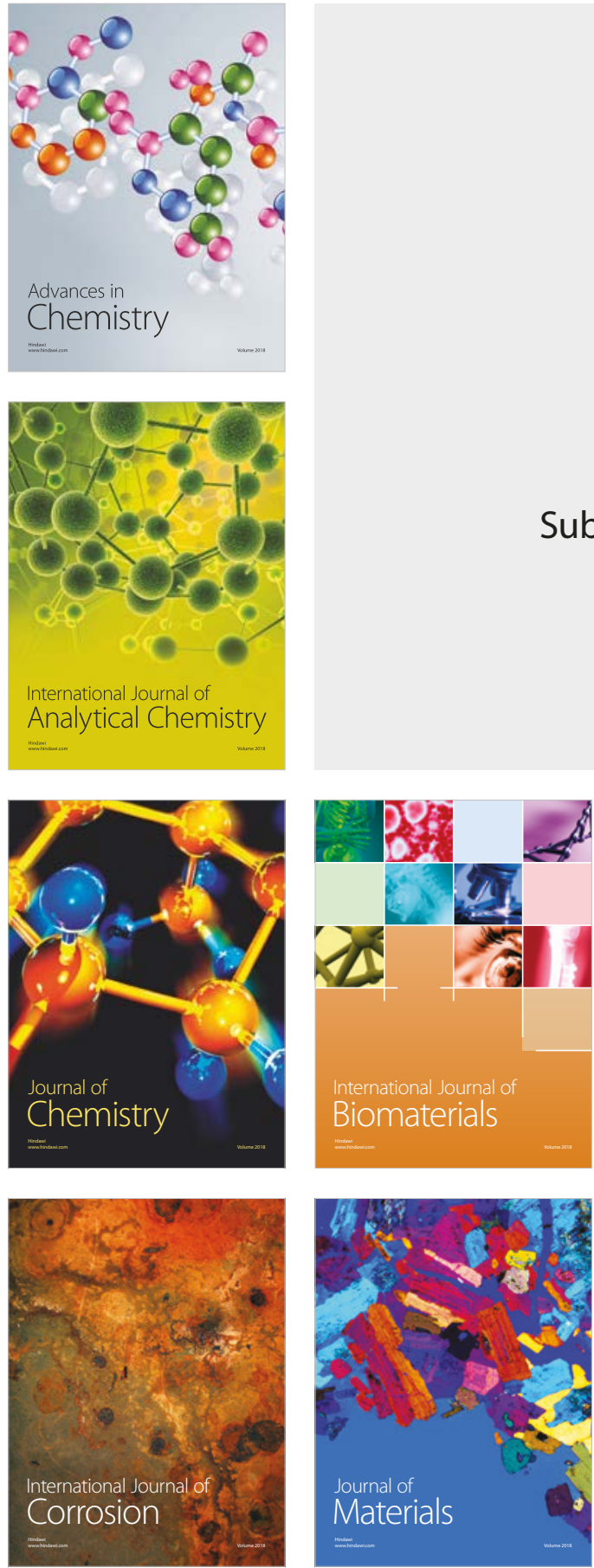

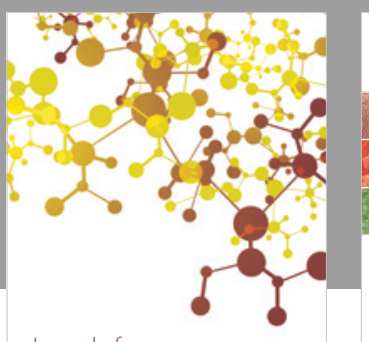

Journal of

Applied Chemistry
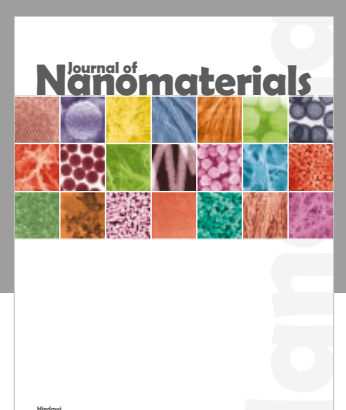

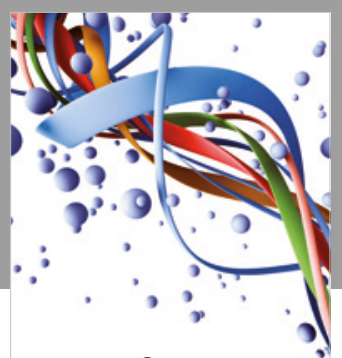

Scientifica

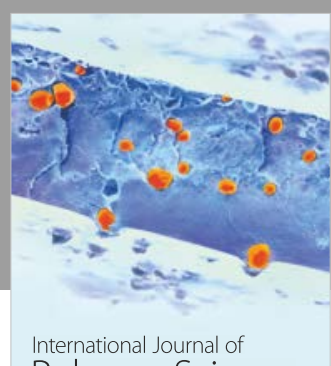

Polymer Science

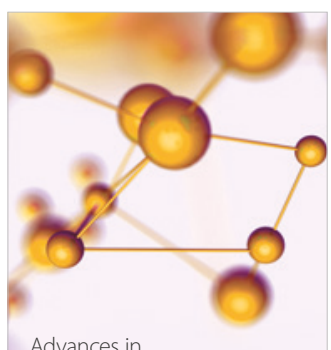

Physical Chemistry
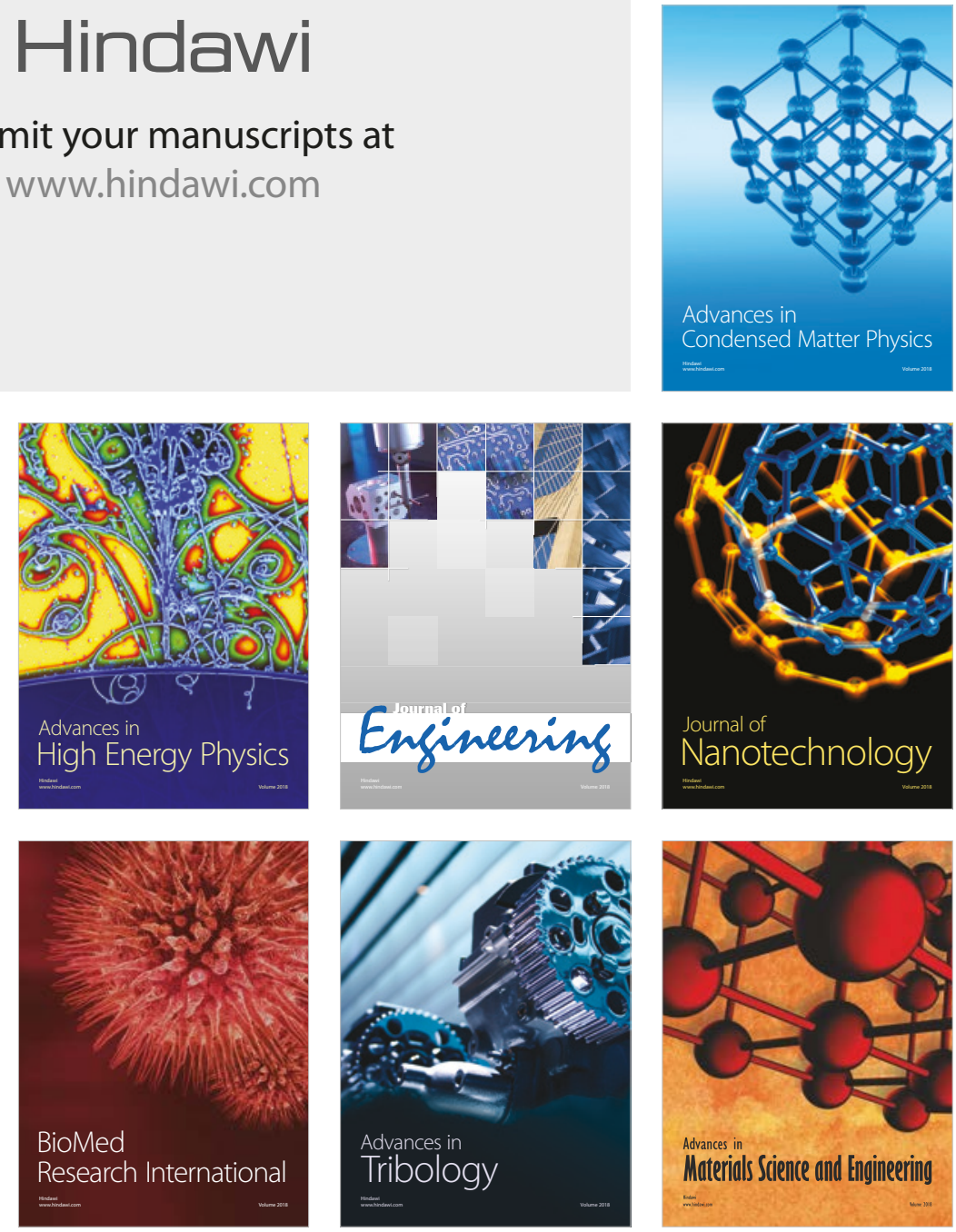